\title{
DISAGREEMENTS BETWEEN GRADIENT-DIFFUSION AND LAGRANGIAN STOCHASTIC DISPERSION MODELS, EVEN FOR SOURCES NEAR THE GROUND
}

\author{
(Research Note) \\ C. J. MOONEY and J. D. WILSON \\ University of Alberta, Edmonton, Alberta T6G 2H4, Canada
}

(Received in final form 15 July, 1992)

\begin{abstract}
It is well known that if turbulent mass convection is modelled as diffusion, errors result unless trajectories from the source (at $h$ ) to the point of observation $\left(z_{p}\right)$ comprise many statisticallyindependent segments (Taylor, 1921). We show that this is not guaranteed merely by the Lagrangian timescale $(\tau)$ at the source being small (e.g., source at ground), but that a better criterion is $t \gg \max \left[\tau(h), \tau\left(z_{p}\right)\right]$, where $t$ is a typical travel time to $z_{p}$.
\end{abstract}

\section{Introduction}

This note concerns the validity of the gradient-diffusion closure method ( $K$-theory) for calculating vertical $(z)$ dispersion from near-ground sources. It has usually been considered that if the Lagrangian timescale $\tau(h)$ at the source height $(h)$ is very small (i.e., the source is near or at the ground), diffusive solutions ( $K$-theory) should be adequate because one expects that travel times $(t)$ from the source to any realisable observation point should satisfy $t / \tau(h) \geqslant 1$ (e.g., Durbin, 1983). We show here, however, that even in such a case, predictions of $K$-theory are incorrect, our criterion being that they differ from the prediction of the corresponding Lagrangian statistical model (we do not claim that the Lagrangian model is correct, but simply that it is a better model than $K$-theory). Near the ground, both models give predictions in good agreement with observations, and differences between them are a small fraction of the mean concentration. But in the upper region of the plume (where the concentration is admittedly small), the fractional difference is large. Thus apparently it is necessary to impose a more stringent criterion for the acceptability of $K$-theory, namely that $t / \tau\left(z_{p}\right) \geqslant 1$, where $t$ is a typical travel time to the observation point $(P)$ and $\tau\left(z_{p}\right)$ is the timescale at that observation point.

To show that $K$-theory is inadequate, we compare an analytical eddy-diffusion solution with the corresponding Lagrangian stochastic (LS) solution. The LS model implements the same wind profile and boundary conditions as the eddy-diffusion solution, and a vertical velocity variance $\sigma_{w}^{2}$ and a Lagrangian timescale profile that together satisfy $\sigma_{w}^{2} \tau(z)=K$. Any number of earlier-provided $K$-theory solutions (for arbitrary wind and diffusivity) would suffice for our purpose. But for 
the sake of novelty, we present a simple series solution for a continuous elevated line source in the neutral surface layer which treats correctly the non-diffusive near field, and therefore overcomes what is usually regarded as the most serious flaw of $K$-theory.

\section{Eddy-Dispersion and Lagrangian Stochastic Models}

2.1. "ANALYTIC" EDDY-DISPERSION MODEL

The "advection-diffusion" equation

$$
\bar{u} \frac{\partial \bar{c}}{\partial x}=\frac{\partial}{\partial z}\left(K \frac{\partial \bar{c}}{\partial z}\right)
$$

is a simplification of the (averaged) mass conservation equation. We specify wind and diffusivity profiles

$$
\bar{u}=U_{H}\left(\frac{z}{H}\right)^{m}, \quad K=b u_{*} z
$$

and ensure correct windspeed and shear at the arbitrary reference height $H$ by choosing $m=1 / \ln \left(H / z_{0}\right)$, where $z_{0}$ is the surface roughness length. We choose $b=0.65$ as the "von Karman constant for mass." This is controversial, but has been shown by Wilson (1982) and others to yield best agreement with dispersion experiments (in any case, the choice does not affect our conclusions).

Let a continuous line source of strength $Q$ lie at $(x, z)=(0, h)$. We wish to solve the advection-diffusion equation for $x \geqslant 0$ under the inflow condition:

$$
\bar{u}(z) \bar{c}(0, z)=Q \delta(z-h)
$$

with reflection or absorption at $z=z_{0}$. We treat near-field effects (Taylor, 1921) by writing a corrected diffusivity

$$
K^{\prime}(x, z)=K(z)(1-\exp (-x / L))
$$

where $L=\bar{u}(h) \tau(h)$ is the alongwind lengthscale across which the diffusivity achieves its "far-field" value. (Implicitly we are assuming the equivalence $x=$ $\bar{u}(h) t$ for the displacement of the scalar at short times after release; this method has been used previously by Raupach and Legg (1983) and Sullivan and Yip (1989), and is readily shown to yield Taylor's solution for dispersion in homogeneous turbulence.)

The differential operator

$$
\frac{1}{z^{m}} \frac{\partial}{\partial z}\left(z \frac{\partial}{\partial z}\right)
$$


has eigenfunctions

$$
\theta_{n}(z)=\left[J_{0}\left(\frac{\lambda_{n}}{\gamma} z^{\gamma}\right)-\mu_{n} N_{0}\left(\frac{\lambda_{n}}{\gamma} z^{\gamma}\right)\right] / I_{n}
$$

corresponding to eigenvalues $\lambda_{n}^{2}$ (Gradshteyn and Ryzhik, 1980) where $\gamma=$ $(1+m) / 2$, and $J_{0}$ and $N_{0}$ are zeroth-order Bessel functions of the first and second kind (for which simple polynomial formulae are available (Abramowitz and Stegun, 1970)). $\mu_{n}$ can be chosen to enforce a reflection or an absorption boundary condition at $z_{0}$. With suitable specification of the normalising constant $I_{n}$ (for which an expression is given by Abramowitz and Stegun), these eigenfunctions are orthonormal on the range $\left(z_{0}, \delta\right)$ and form a basis for continuous functions of $z$ on that range. $\delta$ is a depth chosen to be large with respect to plume depth. We select a discrete spectrum of eigenvalues by enforcing $\theta_{n}(\delta)=0$. The solution to this transcendental equation may be found by a simple numerical search, which produces a monotonically increasing set of $\lambda$ 's.

By substituting into the governing equation the assumed solution

$$
\bar{c}(x, z)=\sum_{n=1}^{\infty} R_{n}(x) \theta_{n}(z)
$$

then multiplying through by $z^{m} \theta_{p}(z)$ and integrating across the range $\left(z_{0}, \delta\right)$, we obtain with the aid of our inflow condition the specification:

$$
R_{n}(x)=\left(\frac{Q h^{m} \theta_{n}(h)}{\bar{u}(h)}\right) \exp \left[\left(\frac{-\lambda_{n}^{2} H^{m} b u_{*}}{U_{H}}\right)\left(x-L+L e^{-x / L}\right)\right] .
$$

\subsection{LAGRANGIAN STOCHASTIC MODEL}

We compare the above analytical solution with the prediction of a Lagrangian stochastic (LS) model of the vertical motion that, for inhomogeneous turbulence having Gaussian velocity distribution $g_{a}(z, w)$ with height-independent variance $\sigma_{w}^{2}$, uniquely satisfies Thomson's (1987) "well-mixed" criterion. The fluctuating horizontal velocity $u^{\prime}$ is neglected relative to the mean (which is consistent with the $K$-theory model), and the LS model is defined by:

$$
\begin{aligned}
& \mathrm{d} w=-\frac{\mathrm{d} t}{\tau_{L}} w+\sqrt{\frac{2 \sigma_{w}^{2}}{\tau_{L}}} \mathrm{~d} \xi_{w} \\
& \mathrm{~d} z=w \mathrm{~d} t \\
& \mathrm{~d} x=U_{H}\left(\frac{z}{H}\right)^{m} \mathrm{~d} t \\
& \mathrm{~d} t=\mu \tau_{L}(z) .
\end{aligned}
$$

Here $\mathrm{d} \xi_{w}$ is a random variate chosen from a Gaussian distribution with mean zero 


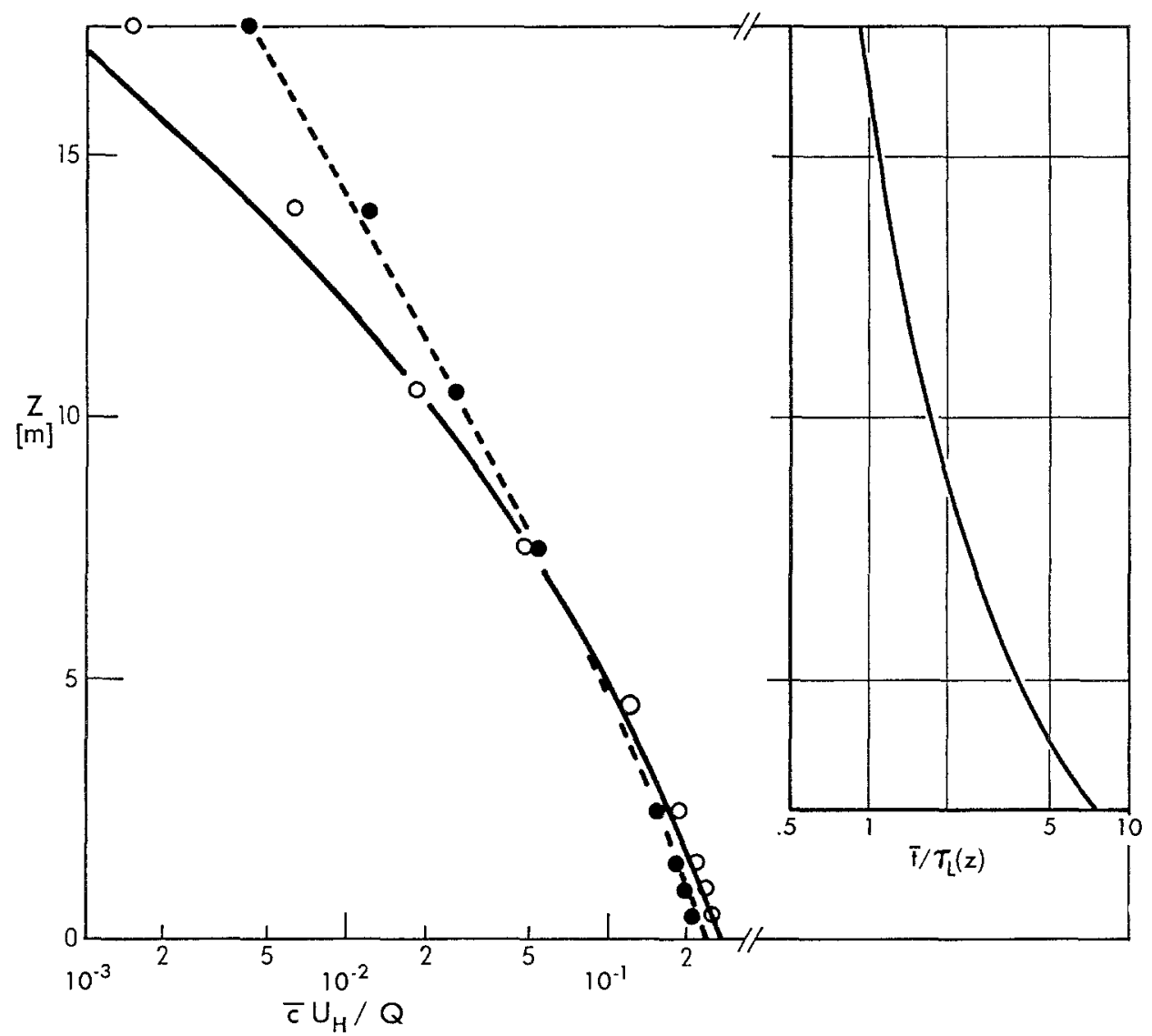

Fig. 1. Comparison of models with the Project Prairie Grass observations of cross-wind integrated concentration at distance $\mathrm{x}=100 \mathrm{~m}$ downstream from a continuous source, and inset, the ratio of mean travel time to the Lagrangian timescale at the point of arrival. ----- Eddy-diffusion solution. Lagrangian stochastic solution. Observations, $\mathrm{L}_{\mathrm{mo}}<-30 \mathrm{~m}$. O Observations, $\left|\mathrm{L}_{\mathrm{mo}}\right|>60$.

and variance $\mathrm{d} t$, and $\mu$ is required to satisfy $\mu \ll 1$. Trajectories are confined to $z \geqslant z_{0}$ by perfect reflection.

\section{Comparison of Models and Observations}

In Figure 1, our $K$-theory and LS solutions are compared with Project Prairie Grass observations (PPG; Barad, 1958) of the vertical profile of normalised mean crosswind-integrated concentration $\bar{u}_{H} \bar{c} / Q$ at a distance $100 \mathrm{~m}$ downstream from a continuous source at height $h=0.46 \mathrm{~m}$. Our simulations used $z_{0}=0.006 \mathrm{~m}$, and we set the reference height $H=10 \mathrm{~m}$ (so both models have correct wind and shear 
at $z=10 \mathrm{~m})$. As the PPG tracer $\left(\mathrm{SO}_{2}\right)$ was not absorbed at the ground, we used the reflection boundary condition.

The $K$-theory and LS solutions both predict well the observed concentration, except at large heights where concentration is anyway small (compared to its value at ground level). At those large heights the two solutions differ by a large factor (though by a small amount in absolute terms), with the $K$-theory concentration being the higher. If the source is placed at the ground, the differences persist. Both solutions necessarily conserve mass.

Before commenting on the discrepancy between the solutions aloft, and the issue of which is "superior," we need to discuss our representation of the "neutral" PPG data. We have plotted both the average of 10 runs that were all performed in slightly unstable stratification (runs 33, 57, 20, 30, 61, 26, 62, 49, 44, 48) and satisfy the criterion on the Monin-Obukhov length that $L_{\mathrm{MO}}<-30 \mathrm{~m}$; and the average of nine runs $(33,57,20,21,42,37,24,38,45)$ that, regardless of stratification, satisfy $\left|L_{\mathrm{MO}}\right|>60 \mathrm{~m}$. The latter representation of "neutral" PPG data yields smaller concentrations far aloft, presumably because a given magnitude for $L_{\mathrm{MO}}$ on the stable side more strongly reduces the eddy diffusivity than the same magnitude on the unstable side increases it. Probably the "unstable runs only" selection is more justifiable. We mention the point because such ambiguity makes it hazardous to try to judge, by the sole criterion of agreement with available atmospheric tracer experiments, which is the more correct of the theoretical solutions.

As can be deduced from the mean travel times $(\bar{t})$ inset on Figure 1, the diffusion and LS solutions agree well only at heights $z$ sufficiently small that $\bar{t} / \tau(z)$ is large. Despite its apparently worse agreement with the $\left(L_{\mathrm{MO}}<-30 \mathrm{~m}\right)$ observations aloft, we regard our LS solution as the more correct of the two (though certainly not as absolutely correct). It derives from clear (but not exactly-correct) assumptions (that the Eulerian velocity probability density function $g_{a}(z, w)$ is Gaussian, and that the particle state $(z, w)$ evolves as a Markov process), and has the weight of Thomson's criteria behind it.

The LS model would have a stronger claim to represent reality if the streamwise velocity fluctuation $u^{\prime}$ and its correlation with $w$ had been included. But the formulation of an unambiguous multi-dimensional LS model, with proper consideration of the implications of (for instance) non-Gaussian velocity distributions and incompressibility, is not at present possible. Furthermore, even when better LS models do become available, the particular model here used will remain the logical standard of comparison for the present $K$-theory solution. It is appropriate because (like the $K$-theory model that it judges) it is one-dimensional, and because it implements trajectories subjected to the minimum restrictions implied by $K$-theory. $K$-theory in its simplest form is but a parameterisation of the net result of stochastic trajectories with velocity scale $\sigma_{w}$ and timescale $\tau_{L}$. (In the same vein, Fick's law of molecular diffusion is only a useful macroscopic law for the outcome of a 
microscopic process whose details are hidden.) There is no room in $K$-theory to address finer points such as the actuality of the velocity pdf's.

\section{Conclusion}

The case of a plume dispersing from a near-ground source over a smooth surface (by "smooth" we do not mean that the surface is aerodynamically smooth, but that the plume is observed at heights greatly exceeding the roughness length $z_{0}$ ) has been regarded as one of the few where $K$-theory is adequate. It will probably not be a surprise to those familiar with the limitations of $K$-theory that even in this "forgiving" case, deficiencies remain - and manifest themselves most noticeably at the upper edge of the plume, where extremal trajectories contribute.

Many solutions already exist that adequately predict the mean concentration near the ground due to sources in the surface layer. Although there is no practical need for better models of mean concentration, there is always justification for greater rigour. It will be difficult to judge further improvements by the criterion of agreement with the available field observations.

\section{Acknowledgements}

The authors acknowledge support from the Natural Sciences and Engineering Research Council of Canada (NSERC) and the Atmospheric Environment Service (AES) of Environment Canada.

\section{References}

Abramowitz, M. and Stegun, I. A.: 1970, Handbook of Mathematical Functions, Dover.

Barad, M. L.: 1958, 'Project Prairie Grass, a Field Program in Diffusion (Vol. II)', Geophysical Research Papers No. 59, Air Force Cambridge Research Center TR-58-235(II). National Technical Information Service NTIS AD-152573.

Durbin, P. A.: 1983, 'Stochastic Differential Equations and Turbulent Dispersion', NASA reference publication 1103 .

Gradshteyn, I. S. and Ryzhik, I. M.: 1980, Table of Integrals, Series, and Products, Academic Press.

Raupach, M. R. and Legg, B. J.: 1983, 'Turbulent Dispersion from an Elevated Line Source: Measurements of Wind-Concentration Moments and Budgets', J. Fluid Mech. 136, 111-137.

Sullivan, P. J. and Yip, H.: 1989, 'Near Source Dispersion of Contaminant from an Elevated LineSource', J. Appl. Math. Phys (ZAMP) 40, 297-299.

Taylor, G. I.: 1921, 'Diffusion by Continuous Movements', Proc. London Math. Soc. Series 2, 20 , 196-212.

Thomson, D. J.: 1987, 'Criteria for the Selection of Stochastic Models of Particle Trajectories in Turbulent Flows', J. Fluid Mech. 180, 529-556.

Wilson, J. D.: 1982, 'An Approximate Analytical Solution to the Diffusion Equation for Short Range Dispersion from a Continuous Ground-Level Source', Boundary-Layer Meteorol. 23, 85-103. 